\title{
Use of the full-thickness resection device (FTRD) to prevent bleeding after colonic polypectomy in a patient with a left ventricular assist device $\square$
}

(이요 $\odot$

\author{
Authors \\ Richard F. Knoop, Hans-Juergen Richer-Schrag, Robert Thimme, Andreas Fischer
}

Institution

University Hospital Freiburg, University of Freiburg, Faculty

of Medicine, Interdisciplinary Gastrointestinal Endoscopy,

Department of Medicine II

submitted 2.3.2017

accepted after revision 29.3.2017

Bibliography

DOI https://doi.org/10.1055/s-0043-111593 |

Endoscopy International Open 2017; 05: E749-E753

(c) Georg Thieme Verlag KG Stuttgart · New York

ISSN 2364-3722

Corresponding author

Dr. med. Richard F. Knoop, MD, University Hospital

Freiburg, Interdisciplinary Gastrointestinal Endoscopy,

Department of Medicine II, Hugstetter Strasse 55,

D-79106 Freiburg, Germany

Fax: +49 $761270-34020$

richard.knoop@uniklinik-freiburg.de

\section{ABSTRACT}

Background and study aims Treatment of colorectal polyps in patients undergoing permanent anticoagulation is associated with a significantly increased risk of peri-interventional bleeding. This specifically applies to polyps $>1 \mathrm{~cm}$ where endoscopic full-thickness resection (EFTR) with the full-thickness-resection device (FTRD) can be taken into consideration as an alternative approach to classical snare polypectomy.

For these indications, EFTR appears to be superior to conventional techniques especially in terms of sufficient hemostasis due to the mechanical tissue compression achieved by the FTRD's integrated Over-The-Scope Clip (OTSC). This is demonstrated in the following case report of a 59-yearold male with a left ventricular assist device (LVAD) implanted due to congestive heart failure. He presented with intermittent hemorrhage from a large polyp in the ascending colon that was successfully treated via EFTR with the FTRD. A video is included.

\section{Introduction}

Recent studies have demonstrated that small colonic polyps can be removed without interrupting anticoagulation [1]. However, this has only been reported for polyps $<1 \mathrm{~cm}[2]$. Management of larger colorectal polyps in patients undergoing permanent anticoagulation remains challenging as they are associated with a high risk of peri-interventional bleeding. Endoscopic full-thickness resection (EFTR) with the full-thickness-resection device (FTRD; Ovesco Endoscopy, Tuebingen, Germany) is increasingly gaining acceptance as an established technique. As presented by our group, FTRD can also be successfully used in order to achieve sufficient prevention of bleeding after colonic polypectomy in a patient with a left ventricular assist device (LVAD) under strict therapeutic anticoagulation.

\section{Case report}

We report on a 59-year-old male with severe heart failure (NYHA IV) due to ischemic cardiomyopathy after acute myocardial infarction. Three years before, he had obtained a left ventricular assist device (LVAD) with a HeartMate II system (Thoratec, Pleasanton, California, United States). Consequently, the patient received permanent therapeutic anticoagulation with Warfarin (INR between 2 and 3 ).

At a cardiological routine check-up he presented with a severely decreased hemoglobin count of $5.8 \mathrm{~g} / \mathrm{dL}$. Erythrocyte concentrates were transfused. Subsequently, esophagogastroduodenoscopy and colonoscopy were performed. Although no acute gastrointestinal bleeding was revealed, a large polyp was detected in the ascending colon as a possible cause for intermittent hemorrhage ( $\triangleright$ Fig.1a, $\triangleright$ Video). Due to the high risk of bleeding, polypectomy was not performed but a watch-andwait strategy was chosen first. However, several episodes of rel- 


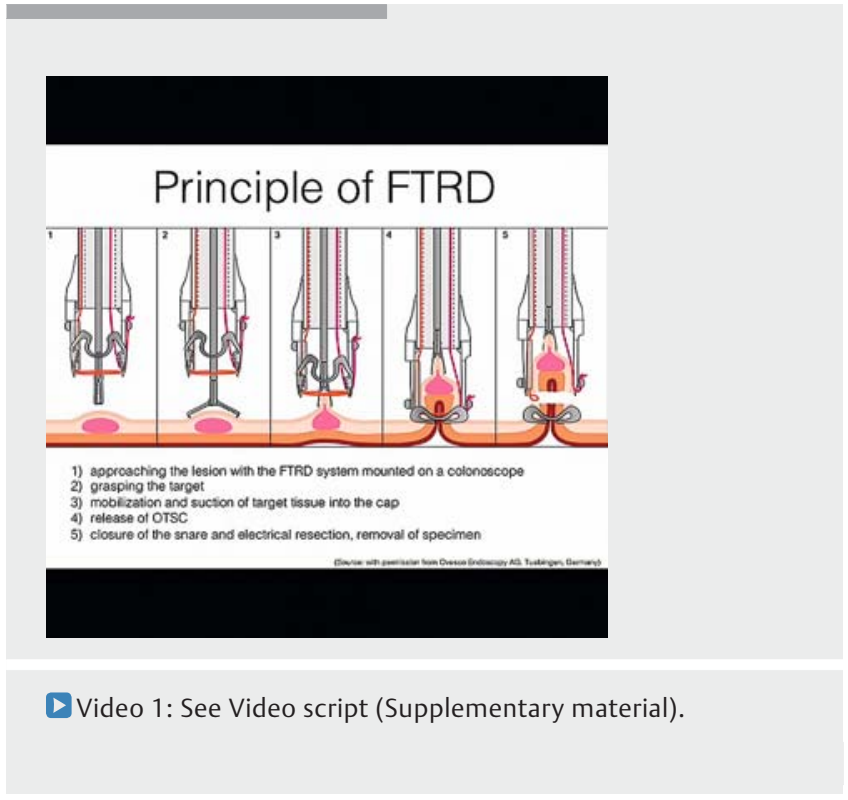

evant rebleeding occurred that required repeated blood transfusions. Therefore, after interdisciplinary reevaluation with all attending physicians, we decided on an interventional endoscopic approach.

Anticoagulation was switched from warfarin to argatroban (Argatra), which was chosen due to the patient's history of heparin-induced thrombocytopenia (HIT).

The polyp was localized behind a colonic fold. This challenged endoscopic evaluation and determination of the polyp's characteristic, e.g. the length of its stalk or base ( $\boldsymbol{F}$ Fig. 1b, - Video). Therefore, even with distal attachment, it was initially not possible to determine whether it contained a very short stalk or a rather flat base, respectively. Under normal conditions, conventional snare polypectomy with facultative hemoclipping and norepinephrine injection therapy (norepinephrine, fibrin glue) would have been an adequate procedure. However, in the past we experienced several significant post-interventional bleeding episodes after comparable snare polypectomies in patients with LVAD with a high risk of hemorrhage. The use of an endoloop might have resolved this problem in cases of polyps with well-defined stalks. However, in the current case of an adenoma with a flat base, that option was not useful. Furthermore, the approach was also abandoned because visualization of the resection site was insufficient. For those reasons, we chose the significantly more invasive procedure of full-thickness resection in this specific case.

Simulation of the resectability with the FTRD was conducted with the FTRD prOVE CAP (Ovesco Endoscopy, Tuebingen, Germany) ( $\triangleright$ Fig. 1c, $\triangleright$ Video). Complete mobilization of the polyp into the transparent cap was achieved. Subsequently, FTRD was successfully performed with surveillance of a cardiology technician and visceral surgery on stand-by in case of an endoscopically unmanageable bleeding.

Histologic examination revealed a full-thickness resection specimen consisting of 2 parts ( $\triangleright$ Fig. 2 a): The polyp itself with $1.5 \times 1.0 \times 0.9 \mathrm{~cm}$ containing a short stalk ( $\vee$ Fig. 2 b) and a colonic wall tissue piece with $3.5 \times 2.5 \times 0.2 \mathrm{~cm}$ ( $\triangleright$ Fig. 2a). Appar- ently, central positioning of the polyp within the specimen was not achieved. Indeed, the colonic wall was aspired into the cap in parallel to the polyp, which resulted in 2 separate resection parts. This was not recognized during the procedure because of the challenging localization of the polyp's base. However, R0 resection was achieved as pathology reported a tubulovillous adenoma with low-grade neoplasia resected in sano (R0).

The next day, endoscopic reevaluation revealed a coagulum at the resection site without active bleeding ( $\triangleright$ Fig. $\mathbf{3 a}, \triangleright$ Video). Prior to discharge 1 week later, colonoscopy confirmed the presence of the FTRD clip without any bleeding stigmata ( $\triangleright$ Fig. 3b, $\triangleright$ Video).

Follow-up colonoscopy 10 weeks later showed a well-healed scar ( $\triangleright$ Fig. 3c, $\triangleright$ Video) while the clip had separated spontaneously. This is congruent with reports in the literature of OTSC detachment within 6 months after resection [3].

\section{Discussion}

The full-thickness resection device is the first commercial system based on the OTSC principle. It has been available for fullthickness colorectal resection since 2014 . The FTRD can be mounted over a standard colonoscope [3]. It is composed of a modified $14 \mathrm{t}$ OTSC on a transparent cap featuring a monofila-

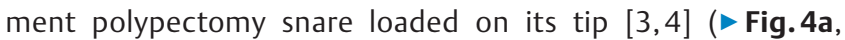
- Video). Because the transparent FTRD cap's volume is greater than that of the OTSC cap $\left(3 \mathrm{~cm}^{3}\right.$ vs. $\left.0.9 \mathrm{~cm}^{3}\right)$, full-thickness specimens with a median resection surface of $5 \mathrm{~cm}^{2}$ (range $\left.1.6 \mathrm{~cm}^{2}-12.9 \mathrm{~cm}^{2}\right)[4,5]$ or a diameter of $3 \mathrm{~cm}$ can be resected, respectively [3] ( $\triangleright$ Fig. 4b, $\triangleright$ Video). The volume of large villous polyps, in particular, may exceed the size of the FTRD cap. In those cases, a simultaneous snare polypectomy with endoscopic full-thickness resection (EFTR) can be performed [5].

Typical indications for FTRD resection are:

- non-lifting lesions

- primary treatment (histology especially regarding resection margin)

- recurrence of adenoma

- secondary treatment due to uncertain R-status

- low-risk early carcinoma

- difficult anatomy (e.g. diverticula, base of appendix)

- subepithelial tumors

Although polypectomy of large colorectal lesions is not a classical indication for EFTR, the procedure was chosen in this patient because it presumably allowed the best compression of the whole resection site and thus prevention of post-interventional bleeding. Hemostasis with conventional techniques such as hemoclipping and injection treatment can fail. In the current case, it was absolutely necessary to avoid emergency surgery. Therefore, EFTR was chosen on the assumption that tissue compression of the whole resection site with the FTRD's modified OTSC is safer than classical polypectomy with subsequent hemostasis. 

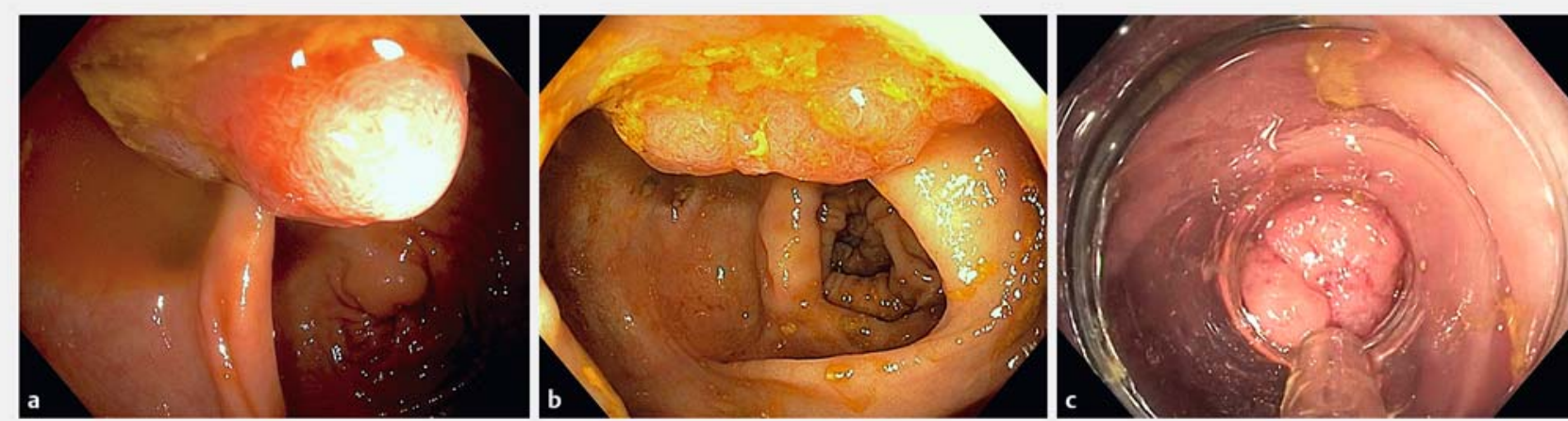

- Fig. 1 a Ascending colon: polyp suspicious for intermittent hemorrhage. b Due to the localization between 2 colonic folds, clear evaluation of the polyp's base (short stalk vs. flat base) was difficult. c Aspiration of the polyp into the FTRD simulation cap (FTRD prOVE CAP, Ovesco Endoscopy, Tuebingen, Germany)
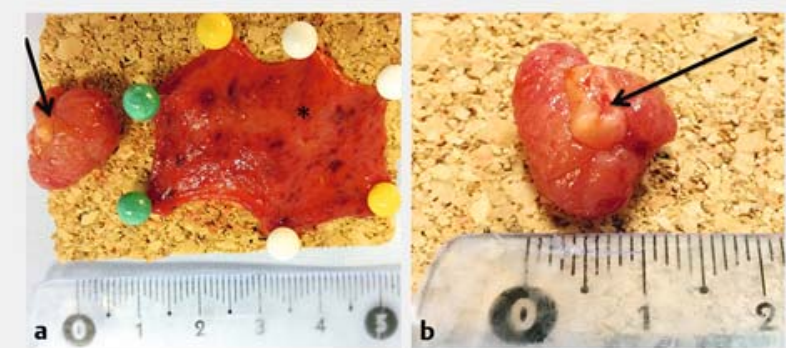

Fig. 2 a, b Full-thickness resection specimen, consisting of 2 parts: the polyp itself with $1.5 \times 1.0 \times 0.9 \mathrm{~cm}$ containing a short stalk (arrows) and a colonic wall tissue piece with $3.5 \times 2.5 \times 0.2 \mathrm{~cm}$ (asterisk). Central positioning of the polyp within the specimen was not achieved. Apparently, the colonic wall was aspirated parallel to the polyp into the cap, which resulted in 2 resection parts. This was not recognized during the procedure because of the challenging visualization of the polyp's base. However, R0 resection was achieved.

\section{Conclusion}

In conclusion, the FTRD is a feasible alternative to classical snare polypectomy for treatment of colorectal polyps of appropriate size. It is particularly relevant to patients who are undergoing permanent anticoagulation and thus at high risk of periinterventional bleeding. Because of the mechanical tissue compression achieved by the OTSC, EFTR appears to be superior to conventional techniques, especially in terms of achievement of sufficient hemostasis.
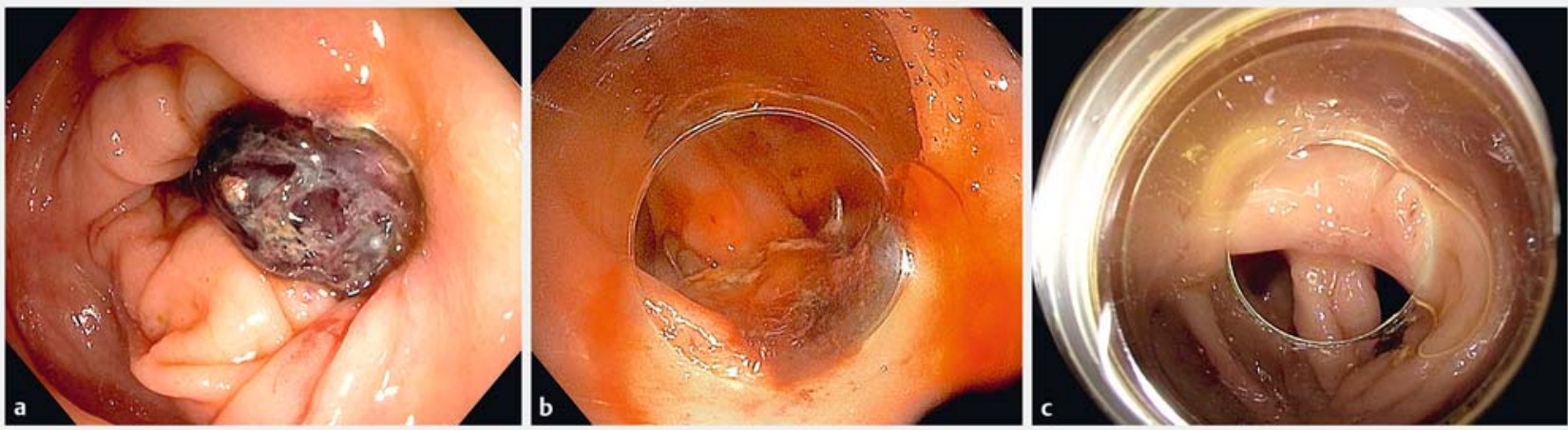

- Fig. 3 a Coagulum at the resection site without active bleeding the day after resection. b Prior to discharge 1 week later: OTSC without any bleeding stigmata. Due to the position between colonic folds, visualization of the latter was only possible using a distance cap (MAJ-663; Olympus, Tokyo, Japan). c Follow-up colonoscopy 10 weeks later: well-healed scar. Apparently, the OTSC was separated spontaneously and excreted with the stool. 


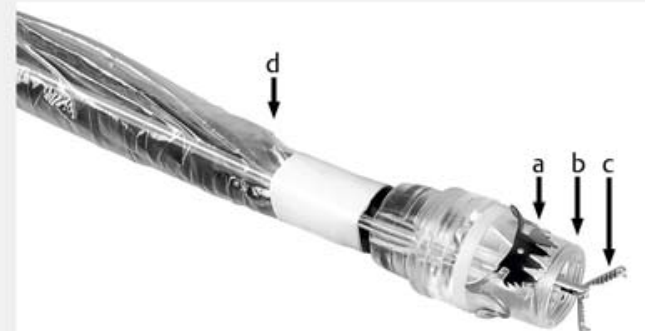

a

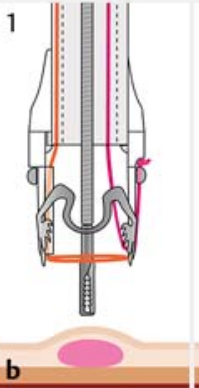

2

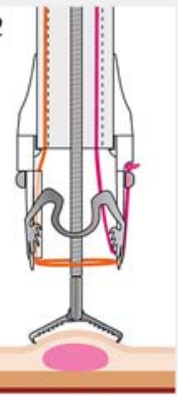

3

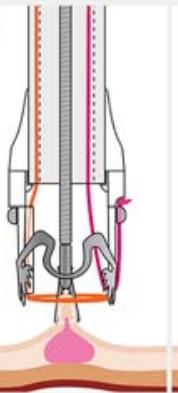

4

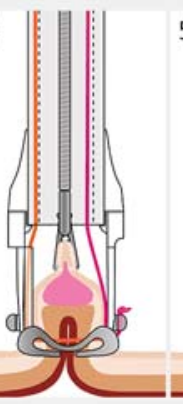

5

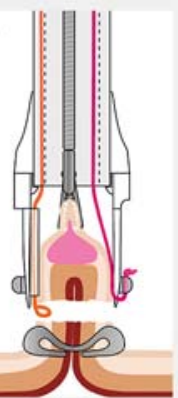

- Fig. 4 a FTRD system with loaded clip and integrated snare mounted on a colonoscope. a Clip. b Snare. c Grasper. d Transparent plastic overlay. b Principle of FTRD: 1) approaching the lesion with the FTRD system mounted on a colonoscope, 2) grasping the target, 3) mobilization and suction of target tissue into the cap, 4) Release of OTSC, 5) closure of the snare and electrical resection, removal of specimen (Source: with permission from Ovesco Endoscopy AG, Tuebingen, Germany)

\section{Competing interests}

None

\section{References}

[1] Kim HG, Friedland S. Safe and effective colon polypectomy in patients receiving uninterrupted anticoagulation: can we do it? Gastrointest Endosc 2014; 79: $424-426$

[2] Horiuchi A, Nakayama Y, Kajiyama M et al. Removal of small colorectal polyps in anticoagulated patients: a prospective randomized com- parison of cold snare and conventional polypectomy. Gastrointest Endosc 2014; 79: 417 -423

[3] Schmidt A, Bauerfeind P, Gubler $C$ et al. Endoscopic full-thickness resection in the colorectum with a novel over-the-scope device: first experience. Endoscopy 2015; 47: 719-725

[4] Richter-Schrag H], Walker C, Thimme R et al. [Full thickness resection device (FTRD) : Experience and outcome for benign neoplasms of the rectum and colon]. Chirurg 2016; 87: $316-325$

[5] Fischer A, Knoop RF, Walker C et al. Resection of a large rectal polyp with the simultaneous combination of snare polypectomy and fullthickness resection device resection. Endoscopy 2015; 47: (Suppl. 01): E607-608 


\section{SUPPLEMENTARY MATERIAL (VIDEO SCRIPT)}

1. Use of the full-thickness resection device (FTRD) to prevent bleeding after colonic polypectomy in a patient with Left Ventricular Assist Device (LVAD)

2. The FTRD system with loaded clip and integrated snare mounted on a colonoscope: a) clip, b) snare, c) grasper, d) transparent plastic overlay

3. The principle of FTRD: 1 ) approaching the lesion with the FTRD system mounted on a colonoscope; 2) grasping the target; 3 ) mobilization and suction of target tissue into the cap; 4) Release of OTSC; 5) closure of the snare and electrical resection, removal of specimen (Source: with permission from Ovesco Endoscopy AG, Tuebingen, Germany).

4. The case

- 59-year-old male with left ventricular assist device (LVAD) due to ischemic cardiomyopathy presented with a decreased hemoglobin count of $5.8 \mathrm{~g} / \mathrm{dL}$

- Large polyp in the ascending colon suspicious for intermittent hemorrhage

- Relevant rebleeding occurred that required repeated blood transfusions

- We experienced several significant post-interventional bleeding episodes after comparable snare polypectomies in patients with LVAD

- $\rightarrow$ more invasive procedure of EFTR was chosen

- Successful EFTR with FTRD

- Sufficient hemostasis in follow-up colonoscopies

5. Ascending colon: polyp suspicious for intermittent hemorrhage. Due to the localization between 2 colonic folds, lear evaluation of the polyp's base (short stalk vs. flat base) was difficult.

6. Aspiration of the polyp into the FTRD simulation cap (FTRD prOVE CAP, Ovesco Endoscopy, Tuebingen, Germany).

7. EFTR with the FTRD.

8. Post-interventional controls.

9. Conclusion

FTRD provides a feasible alternative approach to conventional snare polypectomy for the treatment of colorectal polyps of relevant size. This specifically applies to patients undergoing permanent anticoagulation thus featuring a high peri-interventional bleeding risk. Due to the mechanical tissue compression achieved by the OTSC, EFTR appears to be superior to conventional techniques especially in terms of sufficient hemostasis. 